\title{
Development of a Medical Expert System as an Expert Knowledge Sharing Tool on Diagnosis and Treatment of Hypertension in Pregnancy
}

\author{
J. Gudu, D. Gichoya, P. Nyongesa, and A. Muumbo
}

\begin{abstract}
This paper outlines the development a Medical Expert System for the diagnosis and treatment Hypertension in Pregnancy to be used in the Reproductive Health Division, at Moi Teaching and Referral Hospital in Eldoret, Kenya. The Diagnostic and Treatment Expert System for Hypertension in Pregnancy has so far remained at the testing phase of its life cycle and is yet to be implemented. During the research, it was found that there is an acute shortage of specialist obstetricians in the Reproductive Health Division which implies that there is also scarce expert knowledge on the diagnosis and treatment of Hypertension in Pregnancy, yet the condition continues to kill many women of reproductive age in Kenya, hence the need to develop the Medical Expert System (MES) as an expert knowledge sharing tool to be used by other medical personnel within the Reproductive Health Division who are not specialists in diagnosis and treatment of Hypertension in Pregnancy.
\end{abstract}

Index Terms-Rule based expert system, medical expert system, medical informatics.

\section{INTRODUCTION}

Kenya was identified by the WHO as one of the 57 countries with a critical health workforce shortage [1] with an average of 6,000 doctors [2]. The doctor-patient ratio in Kenya is 1:6,633 in the urban areas, while in the rural areas it is 1: 8,500, which falls short of the 1:600 standard set by the WHO [1]. Hypertension in pregnancy (HIP) is a very common medical problem encountered by the medical personnel, and is the leading cause of maternal deaths at MTRH. Every day at least 2 pregnant women are diagnosed with HIP and in some cases the number goes up to 5 or even more.

\section{BACKGROUND INFORMATION}

The Division currently has a shortage of medical staff. It has 15 specialist obstetricians, 8 registrars, 1 medical officer, 5 medical officer interns, 5 clinical officers and 12 nurses

Manuscript received April 26, 2012; revised July 28, 2012. This work was supported by the Moi Univeristy VLIR Programme.

J. A. Gudu is with the Moi University, Information and Communication Technology Department, (Phone: +254 721890 40, e-mail: jaelgudu@mu.ac.ke, jaelharrup@gmail.com).

D. Gichoya is with the Moi University, School of Information Sciences, Department of Information Technology, (e-mail: dgichoya@mu.ac.ke, hodit@mu.ac.ke).

P. Nyongesa is with the Moi University, School of Medicine, Department of Reproductive Health (e-mail: drpaulnyongesa@yahoo.com).

A. Muumbo is with the Moi University, Director Information and Communication Technology Department (e-mail: amuumbo@mu.ac.ke, muumboalexis@gmail.com). working directly with patients with HIP. 31 of the medical staff in the Division have less than 5 years of experience in handling cases of HIP while only 15 of them, have more than 5 years experience in handling cases of HIP. The sine qua non (essential action or ingredient) of professionalism is specialized knowledge, and not just any sort of specialized knowledge, it is an accumulated and ordered knowledge, built up over time by the experience, analysis, and insight of predecessors in the field [3]. To be an expert in an area or domain, one must have a prolonged or intense experience through practice and education in a particular field and give an example of a shepherd with 50 years of experience tending flocks would be widely recognized as having complete expertise in the use and training of sheep dogs and the care of sheep [4]. Only 15 , out of the 46 medical personnel qualify to be called specialist obstetricians because they have postgraduate degree (Masters) specialising in gynaecology and obstetrics, while the remaining 31 medical personnel are not specialist in obstetric issues. A specialist obstetrician is a physician who has specialized education and training to diagnose and treat women's health concerns like breast cancer, pregnancy and reproductive system conditions. In the Kenyan, an obstetrician specialises in obstetrics and gynaecology by undertaking a Masters degree in Medicine Obstetrics and Gynaecology for 3 years [5] after undergoing a medicine training and attaining a Bachelors Degree in Medicine at any recognised University, and undergoing a one year internship in a hospital [6] and have been licensed to operate by a recognized medical body, such as the Kenya Medical Association.

\section{RESEARCH METHODOLOGY}

The study was carried out using hybrid of qualitative research methodology at the Reproductive Health Division of MTRH, which was chosen using purposive sampling. The main objective of the study was to develop a medical expert system for diagnosing and treatment of HIP; to examine the medical protocols in place at the MTRH; A sample population, which included: specialist obstetricians, registrars, medical officer interns, clinical officers, and nurses, were chosen using stratified sampling. The knowledge elicitation instruments used included: interviews, analysis of documents, observation, and questionnaires.

\section{DEVELOPMENT OF THE SYSTEM}

The system was developed using the Waterman [7] 
approach as the main approach, while the Dokas [8] approach was incorporated especially in phases where Waterman [7] approach was either too general or not specific. The specific phases used in developing the system included: Problem and need identification; Requirement and system analysis; Design or conceptualization; Knowledge acquisition; Formalization or Knowledge Modeling; Development; Testing; Maintenance.

\section{A. Problem and Need Identification}

The main objective of this phase was to identify, characterize, and define the problems the system will be expected to solve. The main problems identified include: Shortage of specialist obstetricians; the other medical staff in the Division needed expert knowledge and guidance, from the specialist obstetricians, on diagnosis and treatment of HIP.

\section{B. Requirements Analysis}

This phase involved getting to know and understand what the users needed the system to do for them and also stipulate what the system needed to function.

\section{1) User requirements}

The users needed a system that could: Automate the medical protocols for diagnosis and treatment of HIP; Offer training facility on the diagnosis and treatment for HIP.

\section{2) System requirements}

The following system hardware, software, and human (end user) skills requirements are recommended so as to get the fastest, most reliable and upgradeable computer system that can diagnose HIP efficiently and reliably. This was categorised as: Hardware requirements (a computer); Software requirements (Operating and application); what is expected of the user to have in order to use the system (such as medical skills and knowledge and basic computer skills).

\section{Design}

The system consists of five main components:

1) The user interface: The user interface runs on a Windows XP platform, running Visual Prolog, Version 7.3 (Personal Edition). Fig. 2 and Fig. 3 give sample screen shots of the user interface

2) The knowledge base: The knowledge in the knowledge base has been organized using IF-THEN rules. The general form of the rules is:

Diagnosis("Patient has hypertension"):-

symptom ("systolic blood pressure is above 140mm $\mathrm{Hg}^{\prime \prime)}$, symptom ("diastolic blood pressure is above 90mm $\mathrm{Hg}^{\prime \prime)}$, symptom ("systolic blood pressure is still above $140 \mathrm{mmHg}$ on consecutive readings"),

symptom ("diastolic blood pressure is still above 90mmHg on consecutive readings"), !.

3) The inference engine: As for the inference Engine, the user provides information about the problem to be solved and the system then attempts to provide insights derived (or inferred) from the knowledge base by examining the facts in the knowledge base [9]. The system uses the forward chaining inference method (the process of starting with the facts and working forward to the conclusions [10].

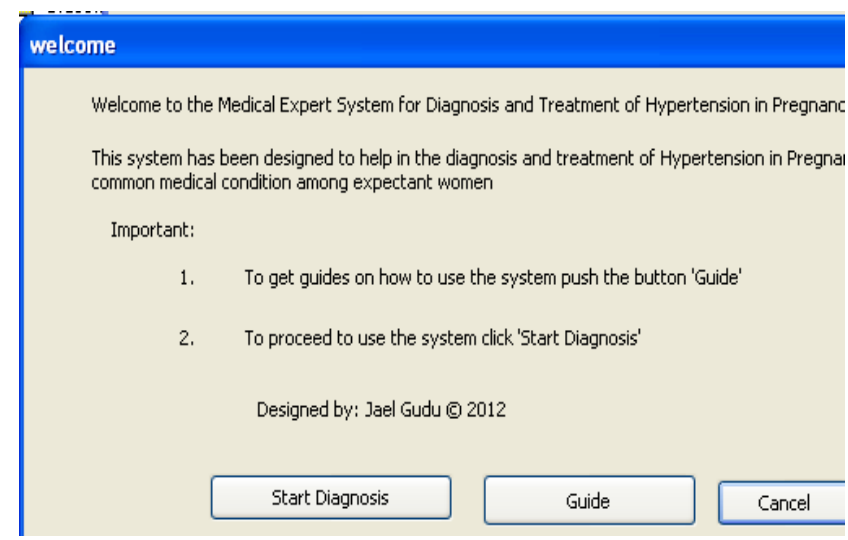

Fig. 1. Sample shot of the systems' start up screen

\section{guide}

GUIDE ON HOW TO USE THE SYSTEM

To start Using the system Click on 'Start Diagnosis' on the Menu Bar.

The steps of diagnosing Hypertension in Pregnancy include:

$$
\begin{aligned}
& \text { 1. Diagnosing Pregnancy } \\
& \text { 2. Diagnosing Hypertension } \\
& \text { 3. Classifying the type of Hypertension that } \\
& \text { 4. Assesing the severity of the type of hype }
\end{aligned}
$$

The dialog box that will show will be for diagnosing Pregnancy

Upon completing the pregnancy diagnosis and the system gives its conclusion tt diagnose Hypertension which will be shown upon reaching the conclusion of the There are also guides on every step of diagnosis on what is expected of the ust

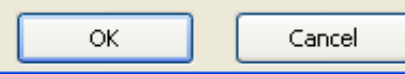

Fig. 2. Sample shot of the systems' start up screen

4) Explanation facility: The explanation facility, on the other hand, allows a user to understand how the expert system arrived at certain results [10].

5) Working memory: Has conclusions reached before they are displayed to the user.

\section{Knowledge Acquisition}

The knowledge acquisition methods used in this study include: interview schedules, analysis of documents and Observation. In some instances, during the knowledge acquisition, the doctors were asked to verbally perform the diagnosis and treatment process loudly, a process known as protocol or task analysis [12]. Some of the questions asked included: What is HIP; How many types of HIP; What are the symptoms of the different types of HIP; How are the different types of HIP diagnosed; How are the different types of HIP treated; What medical rules or protocols guiding the diagnosis and treatment of HIP are in place; How are these medical rules or protocols used and by who.

\section{E. Formalization}

The information and knowledge collected was modeled in the form of decision trees and flow charts to help in understanding how the system is to operate and how it arrives at its conclusion.

Fig. 3 shows the structure of the system showing how the various components, mentioned, work together and was adapted with modifications [11], [12]. 


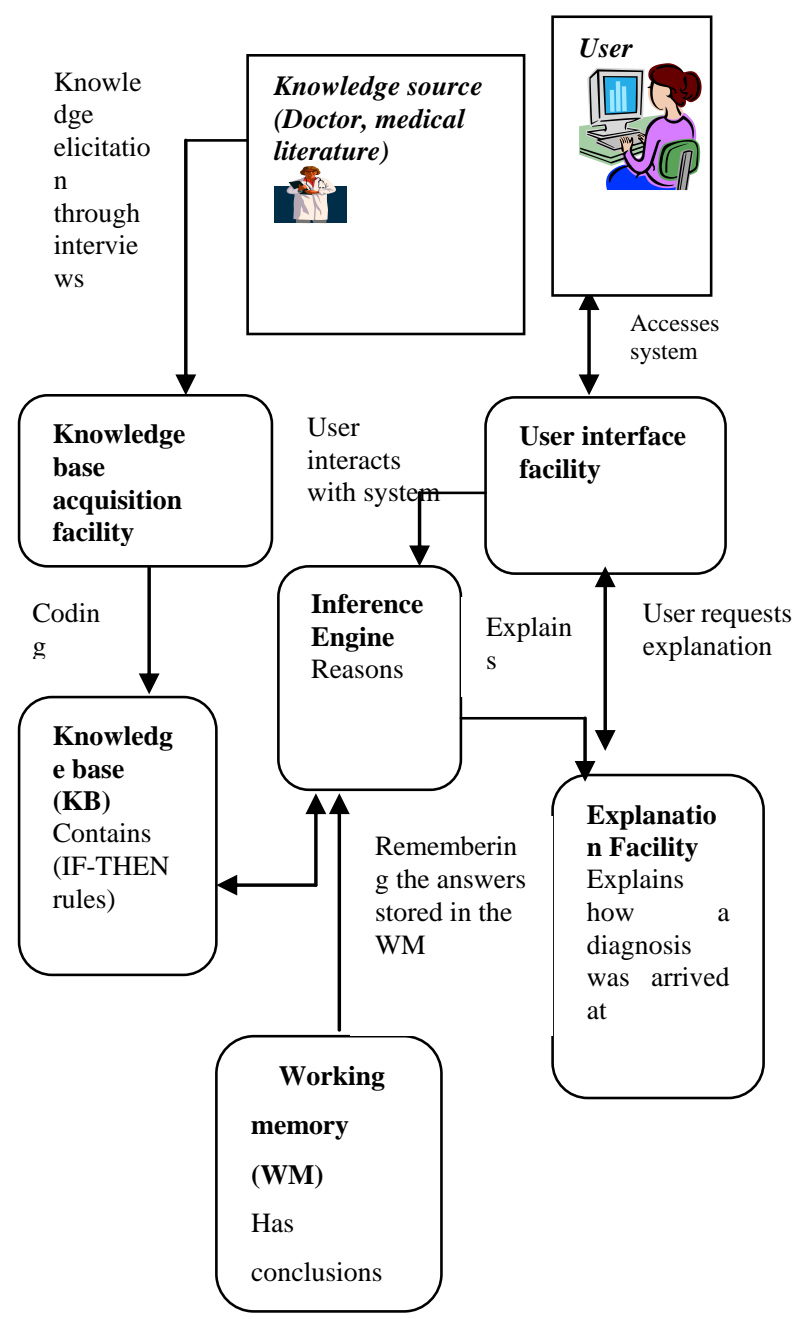

Fig. 3. The structure of the Medical Expert System for HIP

\section{1) How the system works}

The knowledge base of the system contains the facts about the various diseases (preeclampsia, chronic hypertension, gestational hypertension) and what makes each disease what it is (symptoms) that are to be looked out for and the accepted medical protocols to be used to diagnose the disease. For example, if a patient has the following symptoms: patient is pregnant, the pregnancy is below 20 weeks of gestation, evidence of protein in urine (confirmed by a lab test), has a history of hypertension before pregnancy, the blood pressure is equal to or greater than $140 / 100 \mathrm{mmHg}$, and there are signs of fetal distress. What the system does is to go through each of the above stated symptoms by asking the user if the patient is exhibiting any of the symptoms before coming to a conclusion on what the patient is suffering from. If they are all identified to be true the system tells the user that the patient has severe chronic hypertension with superimposed preeclampsia. Since there are other diseases (chronic hypertension, gestational hypertension, Preeclampsia-Eclampsia) in the system that also have their own symptoms to be verified, should the user skips the symptom (meaning that symptom is absent and hence not confirmed to be true) no for an answer to any of the symptoms, the inference engine will quickly jump to the first symptom of the other disease. The system will proceed to check all the other symptoms of that other disease and should they all be positively verified then the system will proceed and give a diagnosis of the correct disease that matches the answers given. Once a diagnosis has been made, the system can either be asked for an explanation as to how the diagnosis was arrived at, and advice on the possible treatment of the diagnosed disease based on accepted medical protocols and algorithms.

\section{F. Development of the Prolog Codes or Commands}

This phase involves the actual coding of the system (writing of the Prolog commands that run the system). The codes were developed and customized in Visual Prolog.

\section{G. Testing and Refinement of the System}

The developed system was put to test in the environment it was expected to operate. The different aspects of the system that were tested included: Relevance: the degree to which the system contained information that the target users applied on a day to day basis when diagnosing and treating Hypertension in Pregnancy; Accuracy: the degree to which the system was correct and on point in delivering its diagnosis on pregnancy, hypertension, classifying the type of hypertension a patient has or could be having, and in specifying the severity or mildness of the type of Hypertension in Pregnancy, and giving treatment advice; Exhaustiveness of the system: the degree to which the system was covering all the necessary areas and steps taken in the diagnosis and treatment of hypertension in pregnancy; Ability to follow the steps of the medical protocol for diagnosis and treatment of Hypertension in Pregnancy: whether or not the system was able to follow the stipulated medical protocol for diagnosis and treatment of Hypertension in Pregnancy; and Ease of use: finding out, from the target users the degree to which they were able to work with the system and get a diagnosis of a patient's condition comfortably. The system was tested on a relatively small population that included two (2) clinical officers and one (1) nurse directly involved in handling patients with HIP. The system was tested on 3 expectant women. Clinical officers and the nurse was selected since the medical expert system for diagnosis and treatment of HIP, would be more useful to these cadre of staff than the specialist obstetricians since they already have the expert knowledge. However, two specialist obstetricians were also involved in the testing of the system. The results were that the system could be used in the intended environment (a hospital) by the target users, but the users had to be trained foremost before they could use the system, but they noted that it was easy to use once the user knew how to operate the system. The system was also found to be: accurate and fast in delivering diagnosis for example the diagnosis of pregnancy shown in Fig. 4; 


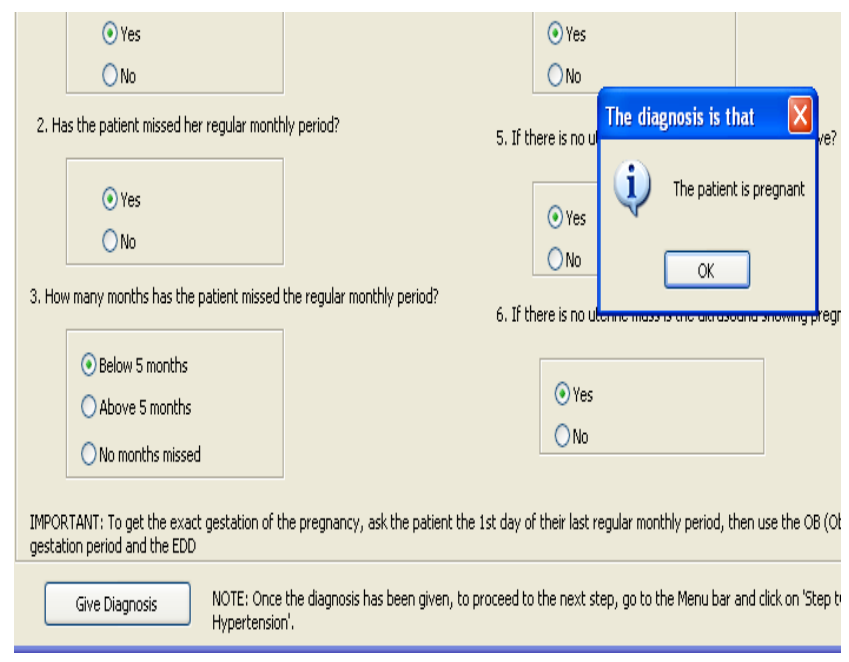

Fig. 4. Sample shot of the systems diagnosing pregnancy.

Relevant as seen in the hypertension knowledge base shown in Fig. 5;

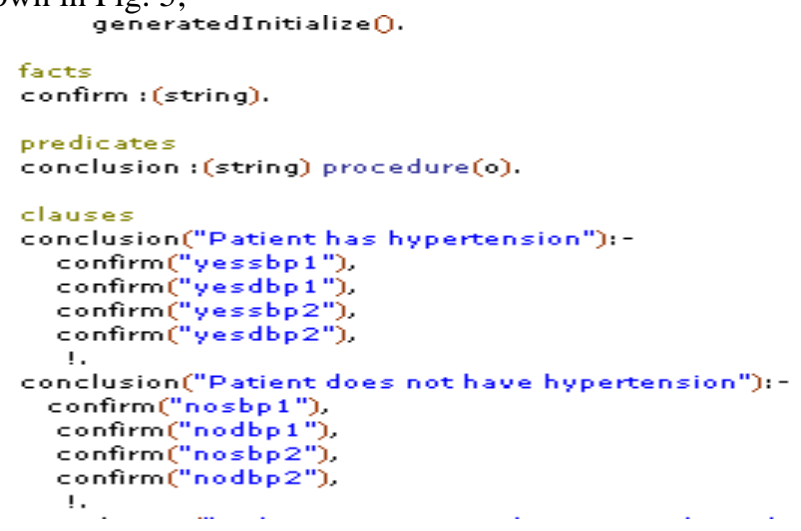

Fig. 5. Sample shot of the hypertension knowledgebase.

Ability to follow the laid down medical protocol results were as follows: on the first test the medical personnel mentioned that the system could not diagnose pregnancy as the first step and moved to diagnose hypertension first, which was not the accepted protocol. However, this concern was addressed and in-cooperated into the system. The system was programmed to first diagnose pregnancy as shown in Fig. 6.

\section{MedicalExpertSsystem

Start Diagnosis File Edit Window Help \\ Step 1: Diagnose Pregnancy \\ Step 2: Diagnose Hypertension \\ Step 3: Classify the type of Hypertension \\ Step 4: Check Severity \\ Step 5: Treatment \\ Step 6: Timing of Delivery \\ Step 7: Method of Delivery}

Fig. 6. Sample shot of the system following the steps of the medical protocol.

\section{H. Maintenance}

Although the medical expert system for diagnosis and treatment of HIP is a conceptually good tool, it is yet to be disseminated to the hospital for use. The system will however be kept current primarily by updating its knowledge base since in healthcare, identification of knowledge quickly becomes a very difficult challenge due to the sheer amount of ever expanding knowledge corpus [13]. It is expected that the medical expert system for diagnosis and treatment of HIP is to be used in different hospitals. This should be a step towards adoption of e-health, and contributing to sharing medical knowledge.

\section{CONCLUSION}

Due to the shortage of specialist obstetricians and the scarcity of the much needed expert knowledge to guide the other medical staff, not yet considered specialists in the field of gynaecology and obstetrics, there was therefore a need to come up with a more effective supportive system for solving this problem characterised by many patients, but few specialists, even if partially, by creating an expert knowledge sharing tool. The Medical expert system (MES) would be handy in sharing the much needed expert knowledge in the diagnosis and treatment of HIP since it would be used by medical officers, clinical officers and nurses in the absence of specialists.

\section{REFERENCES}

[1] WHO, UNICEF, UNFPA, World Bank. Joint statement On Maternal and Newborn Health: Accelerating Efforts to Save the Lives of Women and Newborns, 2008. 11 Nov 2010. [Online]. Available: http://www.unfpa.org/webdav/site/global/shared/safemotherhood/doc s/jointstatement_mnh.pdf

[2] Kenya Medical Association, 2010.

[3] Kennedy and G. Robert, "Why military officers must have training in ethics," University of St Thomas, 2000. 20 Jan 2011. [Online] Available: http://isme.tamu.edu/JSCOPE00/Kennedy00.html

[4] K. A. Ericsson, M. J. Prietula, and E. T. Cokely, "The Making of an Expert," Harvard Business Review, July-August 2007.

[5] University of Nairobi, "Degree Courses: Master of Medicine in Obstetrics and Gynaecology", 2012. 4 Feb 2012. [Online]. Available: http://obsgyn.uonbi.ac.ke/degree_courses/684

[6] EducationPortal.com. "Obstetrician-Gynaecologist Education Requirements: Program Overviews”, 2012. 4 Feb 2012. [Online]. Available:

http://education-portal.com/articles/Obstetrician-Gynecologist_Educat ion_Requirements_Program_Overviews.html.

[7] Waterman, "Donald. A. A guide to expert systems," Addison-Wesley Publishing Company. Massachusetts, 1986.

[8] Dokas and M. Ioannis, "Developing web sites for web based expert systems: A web engineering approach," Proceedings of the Information Technologies in Environmental Engineering (ITEE'2005), pp. 202-217, Germany, 2005.

[9] Griffin, N. L and Lewis F. D (1989), “A Rule-Based Inference Engine which is Optimal and VLSI Implementable". Optimal Inference Engine, 1989. 4 Jan 2011. [Online]. Available: http://www.cs.uky.edu/ lewis/papers/inf-engine.pdf.

[10] Ajith and Abraham, "Rule-based expert systems," Handbook of Measuring System Design, John Wiley and Sons, Ltd. ISBN: 0-470-02143-8, 2005.

[11] M. F. B Zaiyadi, "Expert System for Car Maintenance and Troubleshooting". The Generation5 JDK (Java Development Kit), 2005. 12 Jan 2011. [Online]. Available: http://www.generation5.org/content/2005/CarMaintenance.asp.

[12] M. J. Vlaanderen, "Automated Knowledge Acquisition for Expert Systems: An Overview". Faculty of Philosophy, Erasmus Univerity, Rotterdam, 1990. 6 Jan 2012. [Online]. Available: http://repub.eur.nl/res/pub/16117/AKA_TEKST_9-04.pdf.

[13] BMO A portal for Health Informatics, "Knowledge management: The nature of medical knowledge," 2011. 